\title{
Apatinib plus icotinib in treating advanced non- small cell lung cancer after icotinib treatment failure: a retrospective study
}

This article was published in the following Dove Press journal:

OncoTargets and Therapy

13 October 2017

Number of times this article has been viewed

\author{
Jianping $\mathrm{Xu}$ \\ Xiaoyan Liu \\ Sheng Yang \\ Xiangru Zhang \\ Yuankai Shi
}

Department of Medical Oncology, National Cancer Center/Cancer Hospital, Chinese Academy of Medical Sciences \& Peking Union Medical College, Beijing Key Laboratory of Clinical Study on Anticancer Molecular Targeted Drugs, Beijing, People's Republic of China
Correspondence: Yuankai Shi Department of Medical Oncology, National Cancer Center/Cancer Hospital, Chinese Academy of Medical Sciences \& Peking Union Medical College, Beijing Key Laboratory of Clinical Study on Anticancer Molecular Targeted Drugs, I7 South Panjiayuan Lane, Chaoyang District, Beijing I0002I, People's Republic of China

Tel +86 I0 87788293

Fax +86 I0 87778740

Email syuankai@cicams.ac.cn
Background: Treatment failure frequently occurs in patients with epidermal growth factor receptor (EGFR)-mutant non-small cell lung cancer (NSCLC) who respond to EGFR tyrosine kinase inhibitors initially. This retrospective study tried to investigate the efficacy and safety of apatinib plus icotinib in patients with advanced NSCLC after icotinib treatment failure.

Patients and methods: This study comprised 27 patients with advanced NSCLC who had progressed after icotinib monotherapy. Initially, patients received oral icotinib (125 mg, tid) alone. When the disease progressed, they received icotinib plus apatinib (500 mg, qd, orally). Treatment was continued until disease progression, unacceptable toxicity or consent withdrawal.

Results: Followed up to December 2016, the median time of combined therapy was 7.47 months, and eight of 27 patients were dead. The median overall survival was not reached, and median progression-free survival (PFS) was 5.33 months (95\% CI, 3.63-7.03 months). Moreover, the objective response rate (ORR) was $11.1 \%$, and the disease control rate (DCR) was $81.5 \%$. A total of 14 patients received combined therapy as the second-line treatment, and the ORR and DCR were $7.1 \%$ and $78.6 \%$, respectively; 13 patients received drugs as the third- or later-line treatment, with an ORR and a DCR of $15.4 \%$ and $84.6 \%$, respectively. In addition, 11 patients experienced icotinib monotherapy failure within 6 months with median PFS of 7.37 months, and 16 patients had progression after 6 months with median PFS of 2.60 months. The common drug-related toxic effects were hypertension (44.4\%) and fatigue (37.0\%).

Conclusion: Apatinib plus icotinib is efficacious in treating patients with advanced NSCLC after icotinib treatment failure, with acceptable toxic effects.

Keywords: epidermal growth factor receptor mutation, non-small cell lung cancer, apatinib, icotinib, efficacy

\section{Introduction}

Lung cancer represents one of the most common malignancies and remains the leading cause of cancer-related deaths worldwide. ${ }^{1}$ Non-small cell lung cancer (NSCLC) is the most common subtype of lung cancers, accounting for $85 \%$ of total lung cancer population. ${ }^{2}$ Moreover, patients are usually in advanced stage and not suitable for surgical resection at the time of diagnosis. ${ }^{3}$ Recently, advances in chemotherapy and targeted therapy have provided us with new treatment options for advanced NSCLC.

According to previous statistics, epidermal growth factor receptor (EGFR) gene mutations are present in approximately $17 \%$ of patients with advanced NSCLC, and EGFR-targeted therapy has achieved measurable effects in these patients. ${ }^{4}$ EGFR tyrosine kinase inhibitors (EGFR-TKI) are a group of inhibitors that target EGFR tyrosine kinase. The superior efficacy of EGFR-TKIs for patients harboring 
EGFR-sensitive mutations compared with standard chemotherapy has been demonstrated in several prospective clinical trials. ${ }^{5-7}$ Icotinib, an orally administered EGFR-TKI, showed potent antitumor activity both in vitro and in vivo. ${ }^{8}$ In addition, icotinib has been approved as the first-line therapy in patients with advanced NSCLC with sensitive mutation on February 22, 2011, by China Food and Drug Administration (CFDA). ${ }^{9}$ The efficacy and safety of icotinib in EGFR-mutated patients has been demonstrated in a Phase IV trial. ${ }^{10}$

Although there is a dramatic response to EGFR-TKI initially, the majority of responders will have progressive disease (PD) or even develop acquired resistance. Tumor progression has been reported in patients who previously had been treated with icotinib. ${ }^{11}$ The presence of secondary mutations is a major mechanism contributing to the acquired resistance to the initial EGFR-TKI therapy. Among the secondary mutations, a threonine-to-methionine substitution at amino acid position 790 in exon 20 (T790M) accounts for nearly $50 \%$ of resistant tumors. Moreover, 10\%-20\% of acquired resistance is due to amplification of the c-Met oncogene. ${ }^{12,13}$ Several strategies, such as re-administration with same EGFR-TKI or changes for another, ${ }^{14,15}$ had been tested for treating patients with advanced NSCLC after initial EGFR-TKI failure, while the standard treatment remained to be established.

NSCLC is a highly heterogeneous disease, and multitude of cellular components and patterns of gene expression can affect patient prognosis and response to treatment. ${ }^{16}$ This complicated nature points that multiple signaling pathways and genes are involved in the tumorigenesis and development of NSCLC. Besides EGFR, the vascular endothelial growth factor receptor (VEGFR) is another key molecular target of NSCLC therapy. ${ }^{17}$ To avoid drug resistance and improve efficacy, dual inhibition of both molecular pathways is considered an attractive method due to the following causes. First, EGFR and VEGFR share both parallel and reciprocal downstream signaling pathways, particularly regarding angiogenesis. ${ }^{18}$ VEGF signaling can be influenced by EGFR. ${ }^{19,20}$ Second, since anti-EGFR therapy works on tumor cells and anti-VEGFR therapy works on endothelial cells, dual inhibition of EGFR and VEGFR targets both tumor cells and the endothelial cells, which is supposed to be more effective. ${ }^{21}$ Third, anti-EGFR therapy and antiVEGFR therapy have different toxicity profiles, and the use of these two targeted drugs would potentially have fewer nonspecific toxicities than chemotherapy. ${ }^{22}$ Furthermore, the dual approach against both VEGFR and EGFR signaling pathway has been proposed and showed the efficacy in NSCLC in both preclinical and clinical research. ${ }^{23-25}$ Thus, we supposed that dual inhibition of EGFR and VEGFR may benefit patients with advanced NSCLC who experienced EGFR-TKI treatment failure. Apatinib, a novel small molecule VEGFR inhibitor, has been approved for treating advanced or metastatic chemo-refractory gastric cancer in China. ${ }^{26}$ The beneficial survival of apatinib in NSCLC has also been confirmed in a Phase II clinical trial ${ }^{27}$ that recruited 135 patients with advanced NSCLC after two previous treatment regimens. This retrospective study was designed to determine whether icotinib continuation plus apatinib could bring efficacy benefit for patients with advanced NSCLC after icotinib treatment failure.

\section{Patients and methods} Patients

We retrospectively reviewed the data of patients with advanced NSCLC who received apatinib plus icotinib treatment between February 2014 and June 2016 in Cancer Hospital, Chinese Academy of Medical Sciences. To enroll, patients had 1) pathologically or cytologically confirmed advanced NSCLC, 2) disease progression after icotinib monotherapy, and 3) Eastern Cooperative Oncology Group performance status $($ ECOG PS $) \leq 3$. Patients with ischemic cardiovascular disease, hypertension or proteinuria were excluded from this study.

To preserve patient confidentiality and privacy, patient data have been de-identified before analysis. This retrospective study was performed based on the data from anonymized patients who received apatinib plus icotinib treatment between February 2014 and June 2016. Because of the nature of retrospective design and patient anonymization, the ethics committee of Cancer Hospital, Chinese Academy of Medical Sciences, approved the retrospective study and also determined that informed consent was not required.

\section{Treatment and response evaluation}

Patients received oral icotinib at a dose of $125 \mathrm{mg}$ three times daily, initially. When the disease progressed, patients were continuously treated with icotinib at a dose of $125 \mathrm{mg}$ (tid) plus apatinib at a dose of $500 \mathrm{mg}$ (qd) orally. Treatment was continued until disease progression, unacceptable toxicity or withdrawal of consent.

\section{Patient baseline information}

Patients' demographic and baseline clinical characteristics including sex, age, pathological classification, smoking 
history, tumor stage, ECOG PS, lines of apatinib plus icotinib therapy and EGFR mutation status were collected and analyzed. In addition, hematology, urinalyses, hepatic and renal function tests and contrast-enhanced computed tomography were performed at baseline, a month later after treatment initiation and every 2 months afterward.

\section{Evaluation of treatment response and adverse events}

Objective treatment response was evaluated by computed tomography according to the Response Evaluation Criteria in Solid Tumors (RECIST) version 1.1 and divided into complete remission (CR), partial remission (PR), stable disease (SD) and PD. Progression-free survival (PFS), overall survival (OS), objective response rate (ORR) and disease control rate (DCR) were analyzed. In addition, subgroup analyses were performed based on the line of apatinib plus icotinib treatment as well as the time of icotinib monotherapy failure that patients experienced. Toxicity was assessed by the National Cancer Institute Common Toxicity Criteria (NCI-CTC) version 4.0.

\section{Statistical analysis}

All statistical analyses were conducted using SPSS software version 19.0 (IBM Corporation, Armonk, NY, USA). Categorical variables were presented as percentages and compared using chi-square test. Continuous variables were presented as median (range) and compared using the MannWhitney nonparametric test. PFS was defined as the period between the start of apatinib plus icotinib treatment and the date of documented disease progression or death from any cause, whichever occurs first. OS was defined as the period between the start of apatinib plus icotinib treatment and the date of death from any cause or the most recent date they were known to be alive. DCR was defined as the rate of CR, PR and SD. Median PFS and OS with 95\% CI were estimated using the Kaplan-Meier method. Differences of PFS and OS between two groups were compared using the log-rank test. A $p$-value less than 0.05 was considered statistically significant.

\section{Results \\ Patient characteristics}

Between February 2014 and June 2016, a total of 27 patients with advanced NSCLC after icotinib failure received icotinib in combination with apatinib at our institute. The demographic and baseline clinical characteristics of patients are summarized in Table 1. The median age of the patients was
Table I Patient demographics

\begin{tabular}{ll}
\hline Characteristics & $\mathbf{N}(\%)$ \\
\hline Age (years) & \\
$\quad$ Median (range) & $63(39-79)$ \\
Sex & \\
$\quad$ Male & $12(44.4 \%)$ \\
$\quad$ Female & $15(55.6 \%)$ \\
Smoking history & \\
$\quad$ Never & $24(88.9 \%)$ \\
Former or current & $3(11.1 \%)$ \\
Tumor stage & \\
IV & $27(100 \%)$ \\
ECOG PS & \\
0 & $4(14.8 \%)$ \\
I & $10(37.0 \%)$ \\
2 & $12(44.5 \%)$ \\
3 & $1(3.7 \%)$ \\
Histology & \\
Adenocarcinoma & $23(85.2 \%)$ \\
Squamous cell carcinoma & $3(11.1 \%)$ \\
Large cell carcinoma & $1(3.7 \%)$ \\
EGFR mutation status & \\
Sensitive mutation & $23(85.2 \%)$ \\
Not detected & $4(14.8 \%)$ \\
Line of apatinib plus icotinib treatment & $14(51.9 \%)$ \\
Second-line & $13(48.1 \%)$ \\
Third- or later-line & $11(40.8 \%)$ \\
Time of icotinib monotherapy failure & $16(59.2 \%)$ \\
$>6$ months & \\
\hline Abonths & \\
\hline
\end{tabular}

Abbreviations: ECOG PS, Eastern Cooperative Oncology Group performance status; EGFR, epidermal growth factor receptor.

63 years (range 39-79 years). Most patients were females, accounting for $55.6 \%$. The major histological type was adenocarcinoma in 23 patients (85.2\%). Of 27 patients, 24 had no smoking history. All 27 patients were in stage IV NSCLC. Most patients (51.8\%) had good ECOG PS (0/1). Patients in an ECOG PS of 2 and 3 accounted for $44.5 \%$ and $3.7 \%$, respectively. Of 27 patients, 23 were found with EGFR-sensitive mutation, while in the remaining four patients EGFR mutation was not detected. In addition, 14 of 27 patients $(51.9 \%)$ were treated with icotinib in combination with apatinib as the second-line therapy, and the remaining 13 patients $(48.1 \%)$ were treated with apatinib plus icotinib as the third- or later-line therapy.

\section{Tumor response and survival outcomes}

Followed up to December 2016, the median time of apatinib plus icotinib treatment was 7.47 months. During the follow-up, response was evaluated in all 27 patients. None of 27 patients achieved CR, while three of 27 patients achieved PR, with an ORR of $11.1 \%$. Of 27 patients, 19 had SD, with a DCR of $81.5 \%$, at the best response. In addition, five patients 


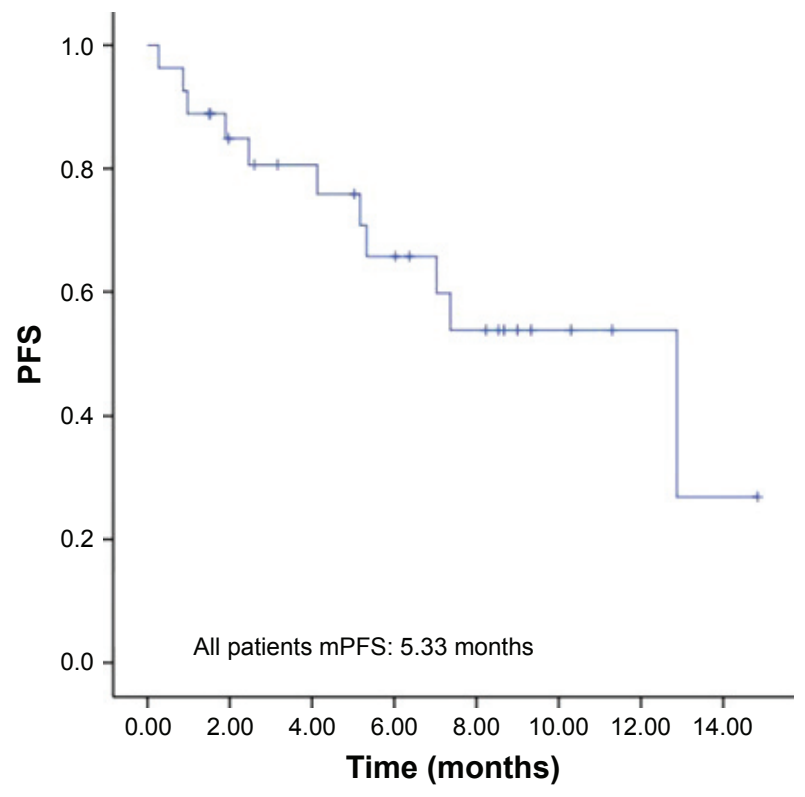

Figure I PFS in 27 patients with advanced NSCLC after apatinib plus icotinib treatment following icotinib monotherapy failure.

Abbreviations: mPFS, median progression-free survival; NSCLC, non-small cell lung cancer; PFS, progression-free survival.

had PD. Eight of 27 patients were dead. The median OS was not reached, and the median PFS was 5.33 months $(95 \% \mathrm{CI}$ 3.63-7.03 months; Figure 1).

In addition, the efficacy of apatinib plus icotinib treatment was also compared between patients treated with these drugs as the second-line therapy and patients treated with these drugs as the third- or later-line therapy. Both median OS and PFS was not reached in 14 patients treated with icotinib in combination with apatinib as the second-line therapy, and the ORR and DCR of these patients were $7.1 \%$ and $78.6 \%$, respectively. Patients treated with apatinib plus icotinib as the third- or later-line therapy had median PFS of 5.33 months (95\% CI 0.27-8.87 months; Figure 2), with an ORR of $15.4 \%$ and DCR of $84.6 \%$. The median OS of these patients was not reached.

Subgroup analyses were also performed based on the time of icotinib monotherapy failure that patients experienced ( $>6$ months vs $\leq 6$ months). The median PFS was 7.37 months (95\% CI 4.17-8.57 months) in 11 patients experiencing icotinib monotherapy failure within 6 months and 2.60 months (95\% CI 0.00-8.54 months) in the remaining 16 patients who experienced icotinib monotherapy failure after more than 6 months (Figure 2). No significant difference was found between them $(p=0.22)$.

\section{Adverse events}

Adverse reactions were evaluated in all enrolled patients, which are summarized in Table 2. The most common adverse event was hypertension in 12 patients (44.4\%). Other common adverse events included fatigue (37.0\%), hepatic injury (29.6\%), anorexia (22.2\%) and hand-foot syndrome $(18.5 \%)$. All these adverse events had been reported before
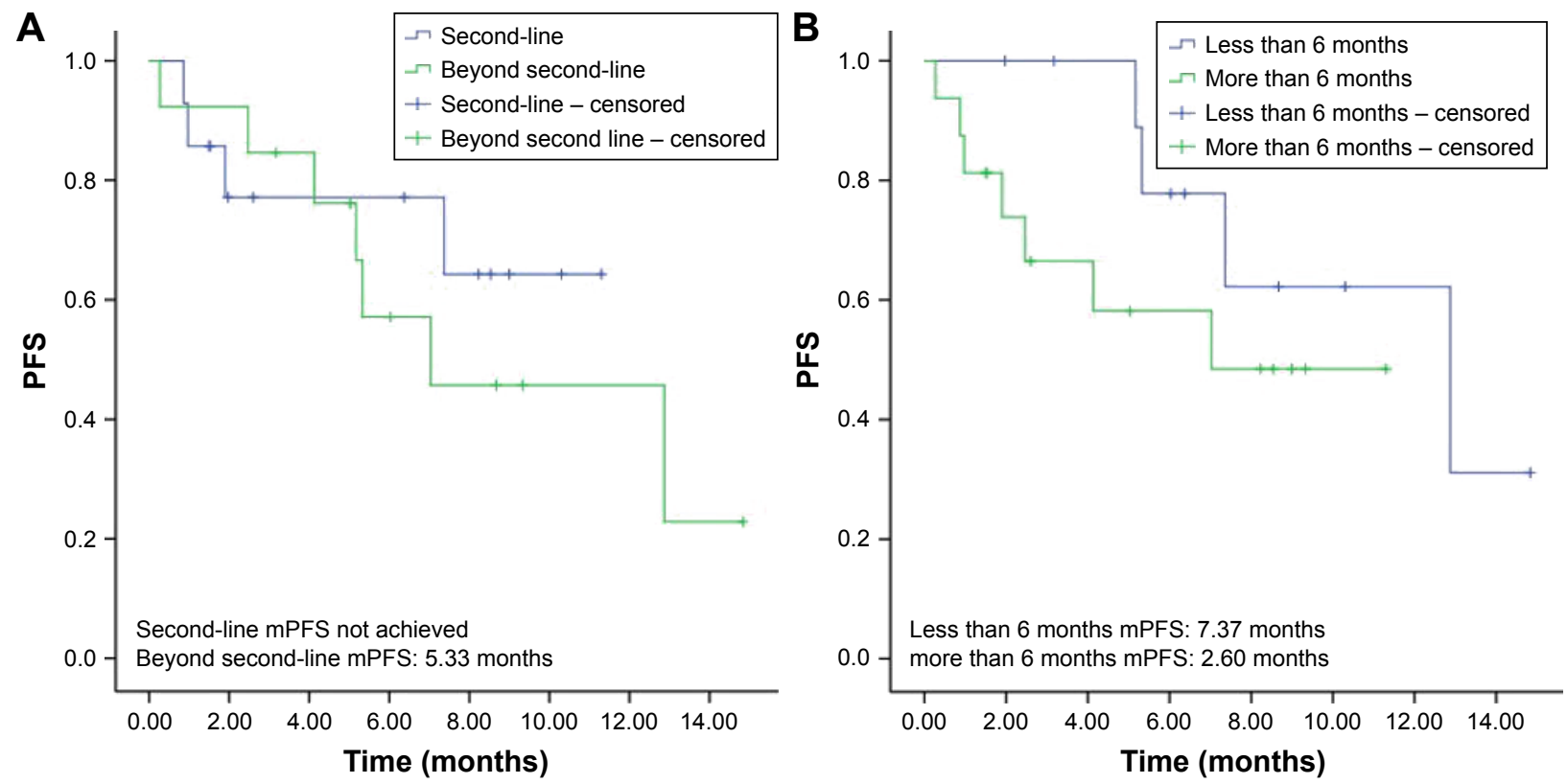

Figure 2 (A) PFS in 14 patients after apatinib plus icotinib treatment as the second-line therapy and in 13 patients after apatinib plus icotinib treatment as the third- or later-line therapy. (B) PFS in II patients who experienced icotinib monotherapy failure within 6 months and in 16 patients who experienced icotinib monotherapy failure after more than 6 months.

Abbreviations: mPFS, median progression-free survival; PFS, progression-free survival. 
Table 2 Adverse events

\begin{tabular}{ll}
\hline Adverse events & N (\%) \\
\hline Hypertension & $12(44.4)$ \\
Fatigue & $10(37.0)$ \\
Hepatic injury & $8(29.6)$ \\
Anorexia & $6(22.2)$ \\
Hand-foot syndrome & $5(18.5)$ \\
Nausea & $3(1 \mathrm{I} .1)$ \\
Palpitation & $\mathrm{I}(3.7)$ \\
Diarrhea & $\mathrm{I}(3.7)$ \\
\hline
\end{tabular}

in the published research of icotinib or apatinib treatment. Furthermore, no grade 3 or 4 adverse event was observed.

\section{Discussion}

In the current study, icotinib re-administration plus apatinib demonstrated an ORR of $11.1 \%$, a DCR of $81.5 \%$ and a median PFS of 5.33 months. The high rate of DCR and long period of PFS demonstrated that icotinib re-administration plus apatinib is efficacious for patients with advanced NSCLC after EGFR-TKI failure. In addition, subgroup analysis indicated that the efficacy of this combination was indistinctive between patients with these drugs as the second-line therapy and those with drugs as the third- or later-line therapy. Although the PFS was higher in patients who underwent icotinib failure within 6 months than those with icotinib failure after more than 6 months, there was no significant difference between them.

In recent years, many efforts have been made on managing the advanced NSCLC after initial EGFR-TKI failure. As recommended by the National Comprehensive Cancer Network (NCCN) guideline, EGFR-TKI including erlotinib, gefitinib or afatinib can be continued in patients after acquiring EGFRTKI resistance who respond to EGFR-TKI initially and do not have multiple systemic symptomatic lesions. ${ }^{28}$ But the efficacies of gefitinib or erlotinib rechallenge alone are moderate, with median PFS of 2.0-3.3 months, ORR of $0 \%-22 \%$ and DCR of $29 \%-67 \% .{ }^{14,29-32}$ Afatinib, a second-generation irreversible EGFR-TKI, has also been investigated as therapy for patients with advanced NSCLC who progressed during prior treatment with other EGFR-TKIs. Based on reported results, afatinib showed modest but not noteworthy efficacy, with median PFS of 4.4 months in Phase II LUX-lung 4 trial and 3.3 months in Phase II/III LUX-lung 1 trial. ${ }^{33,34}$ In addition, the combination of EGFR-TKI with another agent has been evaluated in advanced NSCLC after initial EGFRTKI failure. Cetuximab, an EGFR monoclonal antibody, plus erlotinib showed no significant activity in patients with acquired resistance to erlotinib, with radiographic response of $0 \%$ in a prospective Phase I/II study, ${ }^{35}$ while cetuximab plus afatinib showed a modest efficacy, with median PFS of 4.7 months and ORR of $29 \%$ in a Phase Ib study. ${ }^{36}$ Further study is still needed to confirm the efficacy of cetuximab in combination with afatinib in advanced NSCLC after initial EGFR-TKI failure. Also, it is necessary to explore more effective strategy on managing these patients after initial EGFR-TKI therapy.

Dual blockade of both EGFR and VEGFR pathways is supposed to provide an additive and even synergistic anticancer therapeutic strategy. Both EGFR and VEGFR are critical signaling pathways involved in angiogenesis, and they share several common downstream signaling pathways such as PI3K and MAPK pathway. ${ }^{37}$ The activation of EGFR by EGF can upregulate the production of VEGF in human cancer cells; conversely, EGFR blockade can inhibit the secretion of VEGF and other angiogenic growth factors. ${ }^{19,20}$ As described previously, the combined inhibition of multiple targets has the potential to overcome resistance to monotherapy. ${ }^{38}$ In addition, the dual inhibition of both VEGFR and EGFR has been shown in delaying emergence of resistant tumors in many preclinical and early clinical studies. ${ }^{18,39}$ Based on this anticancer therapeutic strategy, EGFR-TKI rechallenge with bevacizumab in advanced NSCLC, a recombinant humanized monoclonal antibody directed against VEGF, demonstrated a higher DCR of $88 \%$ and modestly longer PFS of 4.1 months (95\% CI 2.3-4.9 months) in a retrospective study. ${ }^{40}$

In the current study, apatinib plus icotinib produced a PFS of 5.33 months in patients with advanced NSCLC, which was longer than other strategies of subsequent treatment after EGFR-TKI failure, such as initial EGFR-TKI readministration, changes for the second-generation EGFR-TKI and continuous EGFR-TKI plus cetuximab or bevacizumab. Apatinib is a small molecule VEGFR-TKI, which binds and strongly inhibits VEGFR-2. Different from bevacizumab that blocks the function of VEGF ligands/receptors extracellularly, apatinib directly targets VEGFR-2 and inhibits VEGFR-2-mediated signaling pathway intracellularly, which may exert broader effects on VEGFR as well as EGFRmediated signaling within the cells. ${ }^{41}$ Apatinib exhibits antitumor activity by targeting endothelial cells. In addition, apatinib has been shown to inhibit tumor cell proliferation and promote apoptosis. ${ }^{42,43}$ These findings may support more PFS benefit of apatinib plus EGFR-TKI in the treatment of advanced NSCLC after initial EGFR-TKI failure.

No treatment-related death occurred during combination treatment. All adverse events were limited to grade 1 or 2 , which were acceptable and manageable. The favorable 
toxicity profile of apatinib in combination with icotinib in the current study could be explained by the prompt and proper treatment on patients once adverse events were present. In case of moderate (not reaching grade 3 or 4 ) adverse events, patients underwent drug suspension or dose modification for drug-related adverse events, or symptomatic treatment for adverse events not related to drugs promptly, resulting in the absence of grade 3 or 4 adverse events.

Furthermore, our study must be faced with several inherent limitations. First, the retrospective design and small sample size inevitably introduce some biases. Second, subgroup analysis has not been performed based on the EGFR mutation status as not all enrolled patients have been detected for EGFR mutation.

\section{Conclusion}

Icotinib continuation plus apatinib revealed a higher DCR and modestly longer PFS than previous description on EGFR-TKI rechallenge alone, changes for another agent or combined with EGFR/VEGFR monoclonal antibodies. In addition, the combination of icotinib and apatinib was well tolerated with acceptable toxicity. Furthermore, the management of adverse events during combination treatment merits more attention.

\section{Acknowledgments}

The abstract of the current study was presented at the 2017 ASCO Annual Meeting. The abstract was published in "Online abstracts" in the Journal of Clinical Oncology (2017;35 Suppl:e20528; http://abstracts.asco.org/199/ AbstView 199 188786). The actual paper, however, has never been published. There was no funding for this study.

\section{Disclosure}

The authors report no conflicts of interest in this work.

\section{References}

1. Siegel RL, Miller KD, Jemal A. Cancer statistics, 2016. CA Cancer J Clin. 2016;66(1):7-30.

2. Travis WD, Brambilla E, Riely GJ. New pathologic classification of lung cancer: relevance for clinical practice and clinical trials. J Clin Oncol. 2013;31(8):992-1001.

3. De Geer A, Eriksson J, Finnern HW. A cross-country review of data collected on non-small cell lung cancer (NSCLC) patients in cancer registries, databases, retrospective and non-randomized prospective studies. J Med Econ. 2013;16(1):134-149.

4. Shigematsu H, Lin L, Takahashi T, et al. Clinical and biological features associated with epidermal growth factor receptor gene mutations in lung cancers. J Natl Cancer Inst. 2005;97(5):339-346.

5. Mitsudomi T, Morita S, Yatabe Y, et al. Gefitinib versus cisplatin plus docetaxel in patients with non-small-cell lung cancer harbouring mutations of the epidermal growth factor receptor (WJTOG3405): an open label, randomised phase 3 trial. Lancet Oncol. 2010;11(2):121-128.
6. Zhou C, Wu Y-L, Chen G, et al. Erlotinib versus chemotherapy as first-line treatment for patients with advanced EGFR mutation-positive non-small-cell lung cancer (OPTIMAL, CTONG-0802): a multicentre, open-label, randomised, phase 3 study. Lancet Oncol. 2011;12(8): 735-742.

7. Sequist LV, Yang JC-H, Yamamoto N, et al. Phase III study of afatinib or cisplatin plus pemetrexed in patients with metastatic lung adenocarcinoma with EGFR mutations. J Clin Oncol. 2013;31(27):3327-3334.

8. Tan F, Shen X, Wang D, et al. Icotinib (BPI-2009H), a novel EGFR tyrosine kinase inhibitor, displays potent efficacy in preclinical studies. Lung Cancer. 2012;76(2):177-182.

9. Shi Y, Sun Y, Yu J, et al. China experts consensus on the diagnosis and treatment of advanced stage primary lung cancer (2016 version). Asia Pac J Clin Oncol. 2017;13(1):87-103.

10. Hu X, Han B, Gu A, et al. A single-arm, multicenter, safety-monitoring, phase IV study of icotinib in treating advanced non-small cell lung cancer (NSCLC). Lung Cancer. 2014;86(2):207-212.

11. Zheng H, Wang Q, Shi H, Zhang H, Hu F, Li B. Favorable response to icotinib in a lung cancer patient with a special mutation at exon 19 of epidermal growth factor receptor. Thorac Cancer. 2014;5(4):358-361.

12. Kobayashi S, Boggon TJ, Dayaram T, et al. EGFR mutation and resistance of non-small-cell lung cancer to gefitinib. $N$ Engl J Med. 2005;352(8):786-792.

13. Oxnard GR, Morris MJ, Hodi FS, et al. When progressive disease does not mean treatment failure: reconsidering the criteria for progression. $J$ Natl Cancer Inst. 2012;104(20):1534-1541.

14. Oh I-J, Ban H-J, Kim K-S, Kim Y-C. Retreatment of gefitinib in patients with non-small-cell lung cancer who previously controlled to gefitinib: a single-arm, open-label, phase II study. Lung Cancer. 2012; 77(1):121-127.

15. Song Z, Yu X, He C, Zhang B, Zhang Y. Re-administration after the failure of gefitinib or erlotinib in patients with advanced non-small cell lung cancer. J Thorac Dis. 2013;5(4):400-405.

16. Chen Z, Fillmore CM, Hammerman PS, Kim CF, Wong K-K. Nonsmall-cell lung cancers: a heterogeneous set of diseases. Nat Rev Cancer. 2014;14(8):535-546.

17. Fontanini G, Vignati S, Boldrini L, et al. Vascular endothelial growth factor is associated with neovascularization and influences progression of non-small cell lung carcinoma. Clin Cancer Res. 1997;3(6):861-865.

18. Tabernero J. The role of VEGF and EGFR inhibition: implications for combining anti-VEGF and anti-EGFR agents. Mol Cancer Res. 2007; 5(3):203-220.

19. Ciardiello F, Caputo R, Damiano V, et al. Antitumor effects of ZD6474, a small molecule vascular endothelial growth factor receptor tyrosine kinase inhibitor, with additional activity against epidermal growth factor receptor tyrosine kinase. Clin Cancer Res. 2003;9(4):1546-1556.

20. Ellis LM. Epidermal growth factor receptor in tumor angiogenesis. Hematol Oncol Clin North Am. 2004;18(5):1007-1021.

21. Dancey JE, Chen HX. Strategies for optimizing combinations of molecularly targeted anticancer agents. Nat Rev Drug Discov. 2006;5(8): 649-659.

22. Sandler A, Herbst R. Combining targeted agents: blocking the epidermal growth factor and vascular endothelial growth factor pathways. Clin Cancer Res. 2006;12(14 pt 2):4421s-4425s.

23. Naumov GN, Nilsson MB, Cascone T, et al. Combined vascular endothelial growth factor receptor and epidermal growth factor receptor (EGFR) blockade inhibits tumor growth in xenograft models of EGFR inhibitor resistance. Clin Cancer Res. 2009;15(10):3484-3494.

24. Ichihara E, Hotta K, Nogami N, et al. Phase II trial of gefitinib in combination with bevacizumab as first-line therapy for advanced non-small cell lung cancer with activating EGFR gene mutations: The Okayama Lung Cancer Study Group Trial 1001. J Thorac Oncol. 2015; 10(3):486-491.

25. Herbst RS, O’Neill VJ, Fehrenbacher L, et al. Phase II study of efficacy and safety of bevacizumab in combination with chemotherapy or erlotinib compared with chemotherapy alone for treatment of recurrent or refractory non small-cell lung cancer. J Clin Oncol. 2007;25(30): 4743-4750. 
26. Li J, Qin S, Xu J, et al. Randomized, double-blind, placebo-controlled phase III trial of apatinib in patients with chemotherapy-refractory advanced or metastatic adenocarcinoma of the stomach or gastroesophageal junction. J Clin Oncol. 2016;34(13):1448-1454.

27. Zhang L, Shi M, Huang C, et al. A Phase II, Multicenter, PlaceboControlled Trial of Apatinib in Patients with Advanced Nonsquamous Non-Small Cell Lung Cancer (NSCLC) after Two Previous Treatment Regimens. Alexandria, VA: American Society of Clinical Oncology; 2012.

28. National Comprehensive Cancer Network, Clinical Practice Guidelines in Oncology (NCCN Guidelines ${ }^{\mathrm{TM}}$ ) [homepage on the Internet]. Non-Small Cell Lung Cancer (version 4.2017); 2017. Available from: http://www.nccn.org. Accessed September 4, 2017.

29. Kaira K, Naito T, Takahashi T, et al. Pooled analysis of the reports of erlotinib after failure of gefitinib for non-small cell lung cancer. Lung Cancer. 2010;68(1):99-104.

30. Asahina H, Oizumi S, Inoue A, et al. Phase II study of gefitinib readministration in patients with advanced non-small cell lung cancer and previous response to gefitinib. Oncology. 2011;79(5-6): 423-429.

31. Hata A, Katakami N, Yoshioka H, et al. Erlotinib after gefitinib failure in relapsed non-small cell lung cancer: clinical benefit with optimal patient selection. Lung Cancer. 2011;74(2):268-273.

32. Koizumi T, Agatsuma T, Ikegami K, et al. Prospective study of gefitinib readministration after chemotherapy in patients with advanced nonsmall-cell lung cancer who previously responded to gefitinib. Clin Lung Cancer. 2012;13(6):458-463.

33. Miller VA, Hirsh V, Cadranel J, et al. Afatinib versus placebo for patients with advanced, metastatic non-small-cell lung cancer after failure of erlotinib, gefitinib, or both, and one or two lines of chemotherapy (LUX-Lung 1): a phase 2b/3 randomised trial. Lancet Oncol. 2012;13(5):528-538.
34. Katakami N, Atagi S, Goto K, et al. LUX-Lung 4: a phase II trial of afatinib in patients with advanced non-small-cell lung cancer who progressed during prior treatment with erlotinib, gefitinib, or both. J Clin Oncol. 2013;31(27):3335-3341.

35. Janjigian YY, Azzoli CG, Krug LM, et al. Phase I/II trial of cetuximab and erlotinib in patients with lung adenocarcinoma and acquired resistance to erlotinib. Clin Cancer Res. 2011;17(8):2521-2527.

36. Janjigian YY, Smit EF, Groen HJ, et al. Dual inhibition of EGFR with afatinib and cetuximab in kinase inhibitor-resistant EGFR-mutant lung cancer with and without T790M mutations. Cancer Discov. 2014; 4(9):1036-1045.

37. Custodio A, Méndez M, Provencio M. Targeted therapies for advanced non-small-cell lung cancer: current status and future implications. Cancer Treat Rev. 2012;38(1):36-53.

38. Rubin BP, Duensing A. Mechanisms of resistance to small molecule kinase inhibition in the treatment of solid tumors. Lab Invest. 2006 86(10):981-986

39. Tonra JR, Deevi DS, Corcoran E, et al. Synergistic antitumor effects of combined epidermal growth factor receptor and vascular endothelial growth factor receptor-2 targeted therapy. Clin Cancer Res. 2006; 12(7):2197-2207

40. Otsuka K, Hata A, Takeshita J, et al. EGFR-TKI rechallenge with bevacizumab in EGFR-mutant non-small cell lung cancer. Cancer Chemother Pharmacol. 2015;76(4):835-841.

41. Imai K, Takaoka A. Comparing antibody and small-molecule therapies for cancer. Nat Rev Cancer. 2006;6(9):714-727.

42. Peng $\mathrm{S}$, Zhang $\mathrm{Y}$, Peng $\mathrm{H}$, et al. Intracellular autocrine VEGF signaling promotes EBDC cell proliferation, which can be inhibited by Apatinib. Cancer Lett. 2016;373(2):193-202.

43. Peng H, Zhang Q, Li J, et al. Apatinib inhibits VEGF signaling and promotes apoptosis in intrahepatic cholangiocarcinoma. Oncotarget 2016;7(13):17220-17229.
OncoTargets and Therapy

\section{Publish your work in this journal}

OncoTargets and Therapy is an international, peer-reviewed, open access journal focusing on the pathological basis of all cancers, potential targets for therapy and treatment protocols employed to improve the management of cancer patients. The journal also focuses on the impact of management programs and new therapeutic agents and protocols on

\section{Dovepress}

patient perspectives such as quality of life, adherence and satisfaction. The manuscript management system is completely online and includes a very quick and fair peer-review system, which is all easy to use. Visit http://www.dovepress.com/testimonials.php to read real quotes from published authors. 